\title{
Paraneoplastic production of heparin-like anticoagulant in a patient with metastatic transitional cell carcinoma
}

\author{
Kelly N. Fahl, BS; Stephen A. Poon, MD; Ketan K. Badani, MD; Mitchell C. Benson, MD
}

\begin{abstract}
It has been reported that patients with bladder cancer have widely varying paraneoplastic consequences, including metabolic, dermatologic, myopathic and neurologic disturbances. We report a case of a 52-year-old man with advanced transitional cell carcinoma and liver metastases, who developed a severe coagulopathy following robotic radical cystoprostatectomy due to circulating heparin-like substances prior to onset of liver failure. Heparin-like anticoagulant production is a rare paraneoplastic effect documented in concert with transitional cell carcinoma, breast carcinoma and hematological malignancies.
\end{abstract}

Can Urol Assoc J 2009;3(5):E61-E63

\section{Introduction}

Paraneoplastic syndromes are rare in bladder cancer, but previous case reports describe a wide range of metabolic, ${ }^{1,2}$ dermatologic, ${ }^{3}$ myopathic ${ }^{4,5}$ and neurological disturbances. ${ }^{6-8}$ Another documented paraneoplastic effect of transitional cell carcinoma (TCC) is the development of coagulopathy from the secretion of heparin-like anticoagulants. ${ }^{9,10}$ We report a case of a patient presenting with this unusual phenomenon.

\section{Case report}

A 52-year-old man with recently diagnosed muscleinvasive bladder cancer during workup for gross hematuria presented to our institution for further management. The patient was without any significant past medical history. He underwent neoadjuvant chemotherapy with 3 cycles of methotrexate, vinblastine, doxorubicin hydrochloride and cisplatin without complication. Restaging computed tomography (CT) scan revealed nodular irregularities of the bladder wall without any pelvic lymphadenopathy. The gallbladder wall was thickened without gallstones or sludge, which was confirmed on ultrasound. Bone scan was negative for metastatic disease. On cystoscopy, nodules of gross tumour were observed at the bladder floor. Repeat transurethral resection revealed high-grade invasive TCC with focal signet features and lymphatic invasion involving the prostatic urethra.
The patient underwent a robotic-assisted laparoscopic cystoprostatectomy, urethrectomy, extended pelvic lymph node dissection and ileal conduit urinary diversion. A cholecystectomy was also performed. Laparoscopy did not reveal any suspicious liver lesions. There was no evidence of gross extravesical disease in the pelvis. The pelvic lymph nodes were found to be sclerotic, but all gross adenopathy was successfully removed. The patient tolerated this operation without complication. His recovery was uneventful except for a superficial infection of his midline incision. The wound was opened and packed.

The patient's surgical pathology returned as poorly differentiated TCC extensively invading perivesical fat, with 20 of 35 pelvic lymph nodes and 2 of 2 pericholecystic lymph nodes positive for metastatic disease (pT3N2). Surgical margins were negative. The patient was discharged on postoperative day 7 , tolerating regular diet with plans to return for further adjuvant treatment.

However, on postoperative day 10, the patient presented to the emergency room with shortness of breath and increased foul-smelling drainage from his midline incision with surrounding cellulitis. The patient was started on empiric anticoagulation with a heparin drip and broad-spectrum intravenous antibiotics. The wound was further opened and the drainage was consistent with an enterocutaneous fistula. Chest x-ray and electrocardiogram were unremarkable. The following day, chest CT angiography showed no evidence of pulmonary emboli or deep venous thrombosis, and the heparin drip was discontinued. After consultation with general surgery, preparations were made to return to the operating room for exploratory laparotomy and fistula take-down. However, on routine repeat laboratory examination on hospital day 7 , the patient had a prothrombin time of 16.2 seconds (international normalized ratio 1.24) and a very elevated partial thromboplastin time (PTT) of 104.6 seconds, despite discontinuation of heparin for 4 days and a dose of enoxaparin 12 hours prior. His liver function tests were grossly normal as prior to initial cystectomy. Surgery was deferred, and despite absence of anticoagulation, his PTT steadily rose over the next several days.

The hematology service was consulted and further laboratory testing was performed. A normal reptilase time despite 
Fahl et al.

PTT $>180$ seconds (Table 1) eliminated dysfibrinogenemia as the cause of the coagulopathy and indicated the presence of heparin. ${ }^{11}$ Serum fibrinogen was normal, indicating that his liver was producing normal amounts of fibrinogen. The patient showed no clinical evidence of bleeding.

Intravenous lines were replaced and parenteral medications discontinued. The patient was placed on close monitoring for 24 hours to ensure that no heparin was administered. Repeat PTT continued to be elevated at $>180$ seconds. As per recommendations of the hematology service, antifactor Xa heparin assay was performed and results were consistent with the therapeutic range for heparin administration. No anti-cardiolipin antibodies were present at the time. Lupus anticoagulant and circulating inhibitor screen tests were unable to be processed, given the presence of heparin in his serum.

On hospital day 11, the patient became progressively jaundiced and liver function tests were suggestive of hepatic dysfunction. Ultrasound revealed an enlarged liver with a diffuse heterogeneous infiltrative process. Follow-up magnetic resonance imaging of the abdomen showed innumerable new liver lesions, consistent with aggressive metastatic disease (Fig. 1). Medical oncology was consulted and the patient's poor prognosis was confirmed. Further treatment was deemed futile and the patient was discharged with home hospice care. The patient expired 2 weeks later.

\section{Discussion}

Heparin is a glycosaminoglycan polysaccharide with a carbohydrate backbone consisting of alternating uronic acid

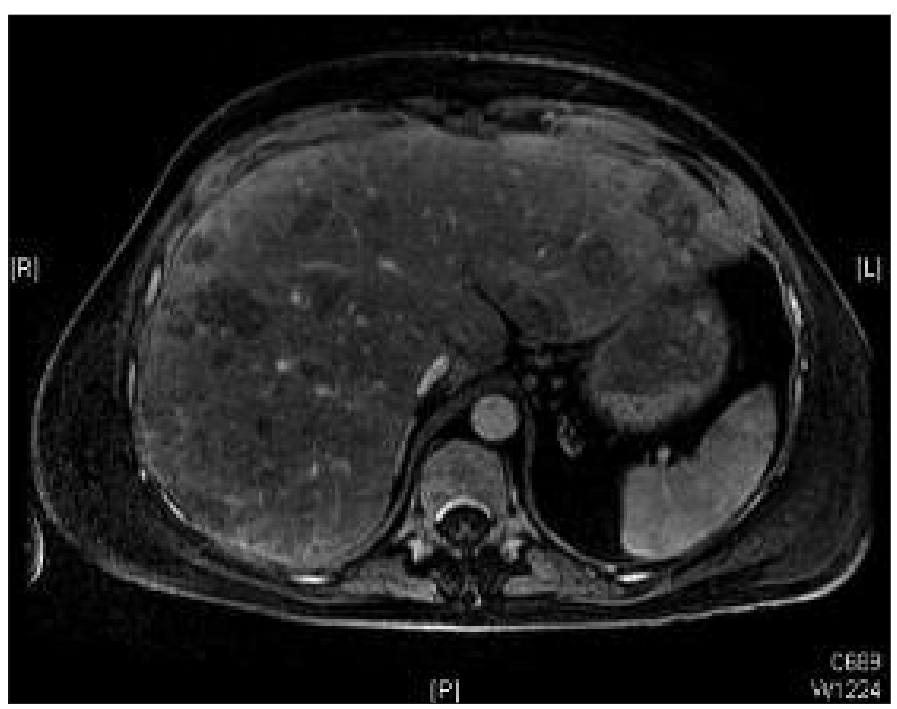

Fig. 1. Gadolinium-enhanced magnetic resonance imaging of the abdomen on hospital day 11 (postoperative day 21) revealing the development of multiple liver metastases.

and hexosamine. It resembles other endogenous glycosaminoglycans, including dermatan sulfate, keratan sulfate and chondroitin sulfate. The phenomenon of endogenously produced heparin-like anticoagulants has been previously described, but is typically associated with hematological malignancies, such as multiple myeloma, chronic lymphoid leukemia, T-cell prolymphocytic leukemia and systemic mastocytosis. ${ }^{12-14}$ Circulating heparin-like activity has been reported in a patient with metastatic adrenocortical carcinoma, undergoing treatment with the anti-protozoal agent suramin, ${ }^{15}$ and also

Table 1. Coagulation studies taken at hospital day 1 of readmission and day 10

\begin{tabular}{lccc}
\hline & Day 1 & Day 10 & Reference range \\
\hline Liver function tests & & & \\
\hline Total protein & 6.7 & 4.5 & $6.7-8.6 \mathrm{~g} / \mathrm{dL}$ \\
\hline Albumin & 4 & 2.2 & $4.1-5.3 \mathrm{~g} / \mathrm{dLhypertension}$ \\
\hline Total bilirubin & 0.5 & 2 & $0.30-1.30 \mathrm{mg} / \mathrm{dL}$ \\
\hline Direct bilirubin & 0.1 & 1.2 & $0.04-0.38 \mathrm{mg} / \mathrm{dL}$ \\
\hline AST & 27 & 205 & $12-38 \mathrm{U} / \mathrm{L}$ \\
\hline ALT & 45 & 59 & $7-41 \mathrm{U} / \mathrm{L}$ \\
\hline Alkaline phosphatase & 71 & $33-96 \mathrm{U} / \mathrm{L}$ \\
\hline Coagulation studies & & 662 & \\
\hline PT & 13.3 & & \\
\hline INR & 0.96 & 21.7 & $12.4-15.0 \mathrm{sec}$ \\
\hline PTT & 36 & 1.79 & $0.88-1.12 \mathrm{units}$ \\
\hline Thrombin time & $\mathrm{N} / \mathrm{A}$ & $23.3-32.3 \mathrm{sec}$ \\
\hline Reptilase time & $\mathrm{N} / \mathrm{A}$ & 180.0 & $15.3-17.9 \mathrm{sec}$ \\
\hline Fibrinogen & $\mathrm{N} / \mathrm{A}$ & $>120.0$ & $16.0-23.6 \mathrm{sec}$ \\
\hline D-dimer & $\mathrm{N} / \mathrm{A}$ & 19.2 & $206-438 \mathrm{mg} / \mathrm{dL}$ \\
\hline Anti-Factor Xa & N/A & 257 & $>0.55 \mathrm{ug} / \mathrm{mL}$ \\
\hline
\end{tabular}

AST=aspartate aminotransferase; $\mathrm{ALT}=$ alanine aminotransferase; $\mathrm{PT}=$ prothrombin time; INR=international normalized ratio; $\mathrm{PTT}=$ partial thromboplastin time; $\mathrm{N} / \mathrm{A}=$ not applicable 
in a woman with metastatic breast cancer. ${ }^{16}$ We were only cable to identify 2 prior cases of heparin-like anticoagulants associated with urothelial cancer. $9,10,14$

Tefferi and colleagues described a 73-year-old woman with metastatic TCC who was found to have circulating heparin-like anticoagulants during workup for vaginal bleeding following radical cystectomy. ${ }^{10,14}$ The patient similarly had a markedly elevated PTT, a slightly prolonged reptilase level, and a positive anti-factor Xa heparin assay. Intravenous protamine was administered with no improvement in bleeding or in in vitro clotting assays. Using affinity chromatography, a substance was isolated with functional and chemical properties similar to high-affinity heparin. Horne and colleagues were able to use plasmapheresis to transiently improve clotting parameters in a patient with advanced TCC and a heparin-like anticoagulant. ${ }^{9} \mathrm{~A}$ serum glycosaminoglycan was isolated with properties of heparin sulfate. Similar to our case, the patient developed extensive liver metastases.

The underlying mechanisms responsible for the production and release of these heparin-like anticoagulants is poorly understood. ${ }^{14}$ In the patient with metastatic adrenocortical carcinoma, damaged liver tissue from suramin was believed to be a possible source of the anticoagulant. ${ }^{15}$ Interestingly, 2 of the 3 reported cases of bladder cancer with endogenous heparin production presented with liver metastases. It is unclear if the presence of heparin-like anticoagulants is associated with metastasis in the liver or with advanced TCC. Regardless, based on the literature, we understand that coagulopathy occurs secondary to production of a new heparin-like glycosaminogly can, either by the tumour, by diseased liver tissue or by the immune system. Therefore, this phenomenon can be considered paraneoplastic rather than the result of liver failure, which would result in coagulopathy secondary to decreased production of clotting factors.

Based on our review, paraneoplastic syndromes are typically associated with aggressive disease. ${ }^{2,17}$ TCC-associated necrotizing polymyositis, ${ }^{5}$ eosinophilic colitis, ${ }^{18}$ and nonislet cell tumour-induced hypoglycaemia ${ }^{1}$ have been described. Neurologic syndromes, including Guillain-Barré and Lambert-Eaton myasthenia, have been associated with bladder cancer. ${ }^{6,8}$

Care for patients with paraneoplastic coagulopathy is largely palliative. The use of protamine is unequivocal and carries the potential risk of anaphylaxis, pulmonary hypertension and bradycardia. ${ }^{19}$ However, protamine should clearly be considered in patients suffering from severe bleeding. ${ }^{13,14,20}$

\section{Conclusion}

The production of circulating heparin-like anticoagulants is a rare paraneoplastic syndrome, but should be considered in patients undergoing treatment for malignancies that result in unexplained coagulopathy. This is more common in patients with haematological malignancies, but as we reported, heparin-like substance production can occur in patients with bladder cancer and may be more common in patients with liver metastases. This syndrome undoubtedly represents advanced underlying disease.

Department of Urology, Columbia University College of Physicians \& Surgeons, New York, NY

This paper has been peer-reviewed.

Competing interests: None declared.

\section{References}

1. Ford-Dunn S, Smith A, Sykes N. Tumour-induced hypoglycaemia. Palliat Med 2002;16:357-8.

2. Hirasawa K, Kitamura T, Oka T, et al. Bladder tumor producing granulocyte colony-stimulating factor and parathyroid hormone related protein. J Urol 2002;167:2130.

3. Lin JT, Wang WH, Yen CC, et al. Prurigo nodularis as initial presentation of metastatic transitional cell carcinoma of the bladder. J Urol 2002;168:631-2.

4. Bouropoulos C, Kanellakopoulou KD, Zarakovitis, IE, et al. Paraneoplastic polymyositis associated with transitional cell carcinoma of the bladder. J Urol 1997;157:950-1.

5. Levin MI, Mozaffar T, Al-Lozi MT, et al. Paraneoplastic necrotizing myopathy: clinical and pathological features. Neurology 1998;50:764-7.

6. Collins DR, Connolly $S$, Burns $M$, et al. Lambert-Eaton myasthenic syndrome in association with transitional cell carcinoma: a previously unrecognized association. Urology 1999;54:162.

7. Greenlee JE, Dalmau J, Lyons T, et al. Association of anti-Yo (type I) antibody with paraneoplastic cerebellar degeneration in the setting of transitional cell carcinoma of the bladder: detection of $Y_{0}$ antigen in tumor tissue and fall in antibody titers following tumor removal. Ann Neurol 1999;45:805-9.

8. Lagrange $E$, Veran 0 , Besson $G$. Pure motor relapsing Guillain-Barre syndrome associated with anti-GM1 antibodies revealing urinary bladder cancer. Eur J Neurol 2007; 14:e7.

9. Horne MK, 3rd, Chao ES, Wilson 0J, et al. A heparin-like anticoagulant as part of global abnormalities of plasma glycosaminoglycans in a patient with transitional cell carcinoma. J Lab Clin Med 1991;118:250-60.

10. Tefferi A, Owen BA, Nichols WL, et al. Isolation of a heparin-like anticoagulant from the plasma of a patient with metastatic bladder carcinoma. Blood 1989;74:252-4.

11. Daniels R: Delmar's guide to laboratory and diagnostic tests. Albany: Delmar Thomson Learning, 2002.

12. Palmer RN, Rick ME, Rick PD, et al. Circulating heparan sulfate anticoagulant in a patient with a fatal bleeding disorder. N Engl J Med 1984;310:1696-9.

13. Sucker C, Mansmann G, Steiner $S$, et al. Fatal bleeding due to a heparin-like anticoagulant in a 37 year-old woman suffering from systemic mastocytosis. Clin Appl Thromb Hemost 2008; 14:360-4.

14. Tefferi A, Nichols WL, Bowie EJ. Circulating heparin-like anticoagulants: report of five consecutive cases and a review. Am J Med 1990;88:184-8.

15. Horne MK, 3rd, Stein CA, LaRocca, RV, et al. Circulating glycosaminoglycan anticoagulants associated with suramin treatment. Blood 1988;71:273-9.

16. Hill GR, Hickton CM, Brennan SO, et al. Acquired heparin-like anticoagulants: a second case in metastatic breast carcinoma and literature review. Clin Lab Haematol 1996;18:291-5.

17. Turalic H, Deamant FD, Reese, JH. Paraneoplastic production of granulocyte colony-stimulating factor in a bladder carcinoma. Scand I Urol Nephrol 2006;40:429-32.

18. Uyar ME, Turkay C, Erbayrak M, et al. Eosinophilic colitis in a patient with advanced transitional cell carcinoma of the bladder: a paraneoplastic syndrome? Am J Med Sci 2008;336:81-3.

19. American Society of Hospital Pharmacists: AHFS drug information. 2008. G. McEvoy, ed. Bethesda, MD: American Society of Hospital Pharmacists.

20. Torjemane L, Guermazi S, Ladeb S, et al. Heparin-like anticoagulant associated with multiple myeloma and neutralized with protamine sulfate. Blood Coagul Fibrinolysis 2007;18:279-81.

Correspondence: Dr. Stephen A. Poon, 161 Ft. Washington Ave., AP-11, Department of Urology, Columbia University College of Physicians \& Surgeons, New York, NY 10032; sap2003@columbia.edu 